\title{
Operational Pattern Analysis for Predictive Maintenance Scheduling of Industrial Systems
}

\author{
Yu Zhang, Chris Bingham, Michael Gallimore, Sepehr Maleki \\ School of Engineering \\ University of Lincoln \\ Lincoln, U.K. \\ \{yzhang; cbingham; mgallimore; smaleki\}@lincoln.ac.uk
}

\begin{abstract}
The paper presents a method to identify the operational usage patterns for industrial systems. Specifically, power measurements from an industrial gas turbine generator are studied. A fast Fourier transform (FFT) and image segmentation is used to develop an intuitive representation of operation. A spectrogram is adopted to study the average usage through the use of spectral power indices, with singular spectral analysis (SSA) applied for operational trend extraction. Through use of these techniques, two fundamental inputs for predictive maintenance scheduling viz. the users behaviour with regard to long-term unit startups patterns, and the duty cycle of power requirements, can be readily identified.
\end{abstract}

Keywords-Operational usage pattern; fast Fourier transform; singular spectral analysis.

\section{INTRODUCTION}

With a view to reducing unit downtime and increasing performance, preventive maintenance planning has received significant attention in the manufacturing and processing industries. An important tool to facilitate these benefits is to accurately analyse operational patterns.

The fast Fourier transform (FFT) has been extensively used in signal processing and machine system analysis for frequency/modal analysis [1], with spectrograms frequently employed for machine fault detection and diagnosis by recognizing fault patterns in the time-frequency domain [2]. Moreover, singular spectrum analysis (SSA) has also been found to be effective for time series analysis and employed for trend extraction and forecasting [3]. Here, FFT, spectrograms and SSA are all used to study the operational pattern of inductrial gas turbines, with basic power measurements being considered as the primary input to the algorithms. The efficacy of the proposed approaches is demonstrated through use of experimental trials on sub-15MW industrial gas turbines.

\section{METHODOLOGY DESCRIPTION}

\section{A. Fast Fourier transform} as

The continuous Fourier transform of signal $f(t)$ is expressed

$$
F(\omega)=\int_{-\infty}^{\infty} f(t) e^{-j \omega t} d t
$$

where $\omega$ is the frequency. Generally, signal is sampled at discrete times $t_{n}$, in which case the discrete Fourier transform (DFT) is expressed as

$$
F(\omega) \approx \sum_{n=1}^{N} f\left(t_{n}\right) e^{-j \omega t}
$$

where $N$ is the total time samples. The FFT is an algorithm to calculate the DFT, which only applies to a signal with a powerof-2 length. It calculates the DFT exactly, but significantly reduces the computation time [4].

\section{B. Spectrogram}

A spectrogram is obtained by first calculating the short time Fourier transform (STFT) [5] of the extracted noise:

$$
\operatorname{STFT}\{x(t)\}(\tau, \omega)=\int_{-\infty}^{\infty} x(t) w(t-\tau) e^{-j \omega t} d t
$$

where $x(t)$ is the original signal, and $w(t)$ is the window function (a Hamming window is used), $\tau$ is a time delay index, and then

$$
\operatorname{spectrogram}(t, \omega)=|\operatorname{STFT}(t, \omega)|^{2},
$$

The spectral power, $S P(t)$, is obtained by integrating the spectrogram wrt. Frequency for each time segment, i.e.

$$
S P(t)=\int_{-\infty}^{\infty} \operatorname{spectrogram}(t, \omega) d \omega,
$$

which can give a further reprehensive illustration of the operational patterns in this case.

\section{Singular spectral analysis}

Consider $x(n), n=1, \ldots, N$, to be a time series. Given the window length $L(1<L<N)$ to be the window length, and $K=$ $N-L+1$. The trajectory matrix of $\mathrm{x}$ with a dimension of $L \times K$ is

$$
\boldsymbol{X}=\left[\begin{array}{cccc}
x_{1} & x_{2} & \cdots & x_{K} \\
x_{2} & x_{3} & \cdots & x_{K+1} \\
\vdots & \vdots & \ddots & \vdots \\
x_{L} & x_{L+1} & \cdots & x_{N}
\end{array}\right]
$$


Set $\boldsymbol{S}=\boldsymbol{X} \boldsymbol{X}^{T}, \lambda_{i}(i=1, \ldots, L)$ are the eigenvalues of $\mathrm{S}$ in a decreasing order. Set $d=\max \left\{j, \lambda_{j}>0\right\}, \boldsymbol{U}_{j}(j=1, \ldots, d)$ are the eigenvectors. The factor vectors are defined as $\boldsymbol{V}_{j}=\boldsymbol{X}^{T} \boldsymbol{U}_{j} / \sqrt{\lambda_{j}}(j=1, \ldots, d)$. Donate $\tilde{\boldsymbol{X}}_{j}=\sqrt{\lambda_{j}} \boldsymbol{U}_{j} \boldsymbol{V}_{j}^{T}$, the singular value decomposition (SVD) of the trajectory matrix is represented by

$$
\boldsymbol{X}=\tilde{\boldsymbol{X}}_{1}+\cdots+\tilde{\boldsymbol{X}}_{d} .
$$

Group the indices set $\{1, \ldots, d\}$ into $m$ disjoint subsets $I_{1}, \cdots, I_{m}$, where each subset has a length $l$. The new expansion of (7) is

$$
\boldsymbol{X}=\tilde{\boldsymbol{X}}_{\boldsymbol{I}_{1}}+\cdots+\tilde{\boldsymbol{X}}_{I_{m}} .
$$

Each matrix $\tilde{\boldsymbol{X}}_{\boldsymbol{I}}$ is hankelized and then the obtained Hankel matrix is transformed into a new series of length $N, \widetilde{\boldsymbol{x}}_{n}^{(k)}$. Finally, the initial time series $x(n)$ can be expressed as a combination of $m$ reconstructed subseries:

$$
x_{n}=\sum_{k=1}^{m} \tilde{\boldsymbol{x}}_{n}^{(k)}(n=1, \ldots, N),
$$

where each subseries could be considered as a part of either trend or noise. By selecting the required frequency components, trend extraction can be obtained from (9).

If the last component $x_{L}$ of any vector $X=\left(x_{1}, \ldots, x_{L}\right)^{T}$, is considered as a linear combination of components $\left(x_{1}, \ldots, x_{L-1}\right), X_{\Delta}: x_{L}=a_{1} x_{L-1}+\cdots+a_{L-1} x_{1}$.

$A=\left(a_{1}, \ldots, a_{L-1}\right)$ is the coefficient vector. The linear operator $\Gamma^{(r)}$ is defined as $\Gamma^{(r)} X=\left(\begin{array}{c}X_{\Delta} \\ A^{T} X_{\Delta}\end{array}\right)$.

By using SSA to forecast $M$ of points, the time series $X_{T+M}=\left(x_{1}, \ldots, x_{T+M}\right)$ is defined as

$$
x_{i}=\left\{\begin{array}{cc}
\tilde{x}_{i} & \text { for } i=1, \ldots, T \\
\sum_{j=1}^{L-1} a_{j} x_{i-j} & \text { for } i=T+1, \ldots, T+M
\end{array} .\right.
$$

Equivalently, it has

$$
Z_{i}=\left\{\begin{array}{cc}
\tilde{X}_{i} & \text { for } i=1, \ldots, K \\
\Gamma^{(r)} Z_{i-1} & \text { for } i=K+1, \ldots, T+M
\end{array}\right.
$$

The matrix $\boldsymbol{Z}=\left[Z_{1}, \ldots, Z_{T+M}\right]$ is the trajectory matrix of the series $X_{T+M}$.

For more details of the SSA algorithm for trend extraction and time series forecasting, readers can be directed to [6].

\section{CASE STUDY}

\section{A. Problem Statement}

This paper considers the operational usage patterns of an industrial generator unit (Fig. 1) as a mechanism to facilitate predictive maintenance scheduling. For convenience in this instance, the operation of the unit is analyzed by considering the generator power measurements. The time period covers 8 years from 2005 to 2012, and the power measurements are collected as hourly 'snapshots', as shown in Fig. 2.

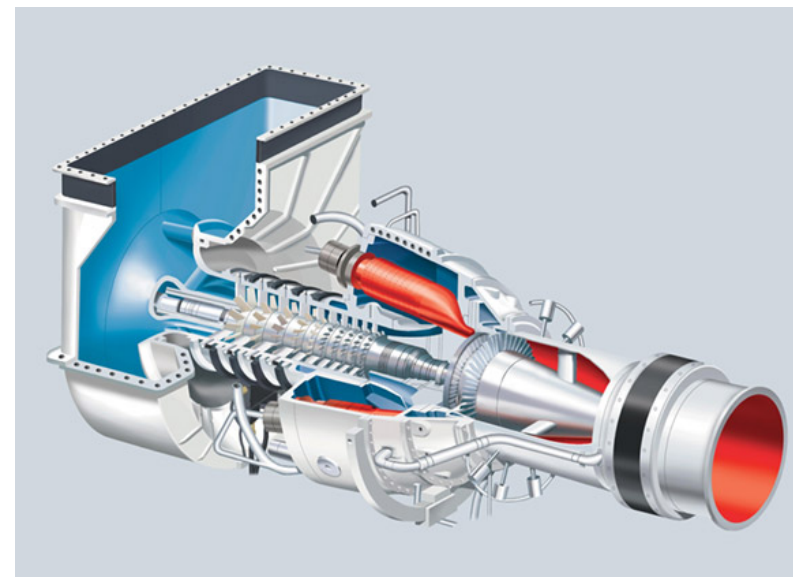

Fig. 1. Schematic of an industrial generator unit.

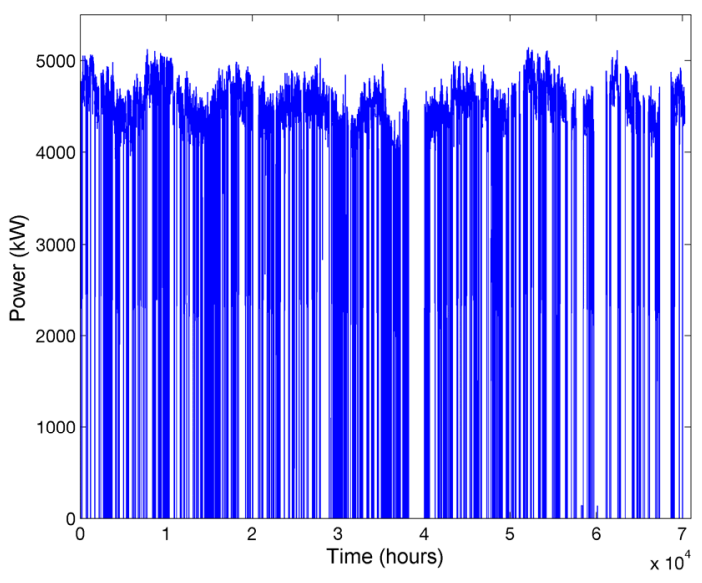

Fig. 2. Power measurements of the unit over a period of 8 years.

\section{B. Frequency/period analysis}

FFT is used to calculate the frequency/period characteristics of the signal in Fig. 2 on the whole record of data, resulting in Fig. 3, where the highest peak is seen to correspond to a period of 8766 hours, i.e. an annual cycle, and the second highest peak locates at a period of 168 hours i.e. a weekly cycle. This can provide important insights of the unit usage information, since further investigations have shown that the primary unit usage is concentrating on weekday daytime during a weekly cycle, and the generator usage is higher in winter period than in summer period during a yearly cycle. 


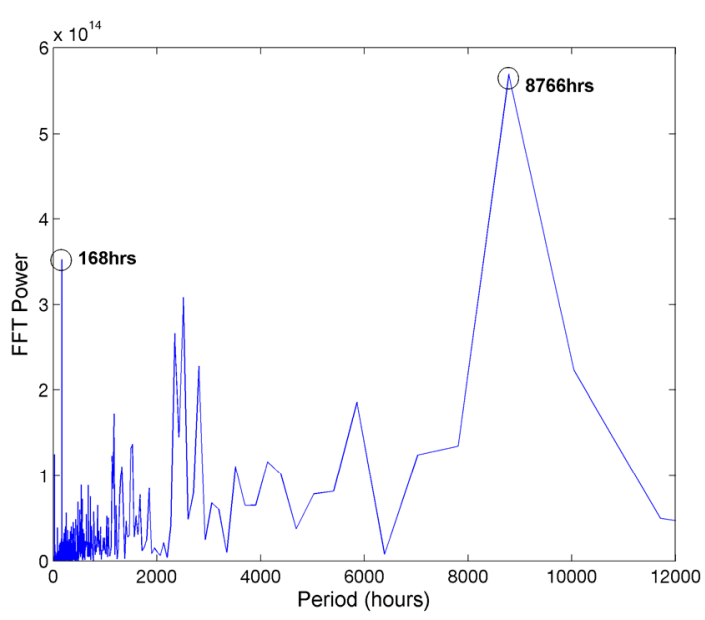

Fig. 3. Periodic operational characteristic

\section{Operational pattern analysis}

By considering the identified weekly operational periods from Fig. 3, the data is rearranged into periods of one week, and the resulting 2D matrix is plotted in Fig. 4, where the $\mathrm{x}$ axis spans 7 days with each day comprising 24 hours (168 points), and the y-axis shows the operation of each week over the full 8 years (416 points). In Fig. 4(a), daily running hours are represented for each day in terms of a color presentation in a cool-hot colour map, where blue represents 0 running hours during that day, and red indicates full running 24 hours. For a more intuitive image presentation, a binary notation: $1=$ unit running (red); $0=$ unit not running (blue), is used in Fig. 4(b), from which, it can be seen that the unit usage is primarily during the daytime on weekdays with shutdown at weekends. This therefore provides a key contributor for identifying the user's startup pattern which is fundamental to predictive maintenance scheduling.

\section{Spectral analysis}

Spectrogram of Fig. 4(b) is now developed, in Fig. 5, where hotter tones represent higher amplitudes of 'energy', and vice versa. It is clearly evident that higher utilization is concentrated during the daytime on weekdays. By integrating the spectrogram energy for each time segment, the spectral power can be calculated. By dividing the computed spectral power by the whole power calculated for total usage, the unit's mean operational usage (percentage) for weekly days can be calculated, as shown in Fig. 6(a).

Similarly, the same methodology has been applied to two other units (Units 2 and 3) at the same site of the studied unit (Unit 1), and the mean percentage usages of the units are plotted in Fig. 6(b). Through Fig. 6(b), the workload of different units can be clearly identified, which can be used as direct numerical input to predictive maintenance scheduling for different generator units.

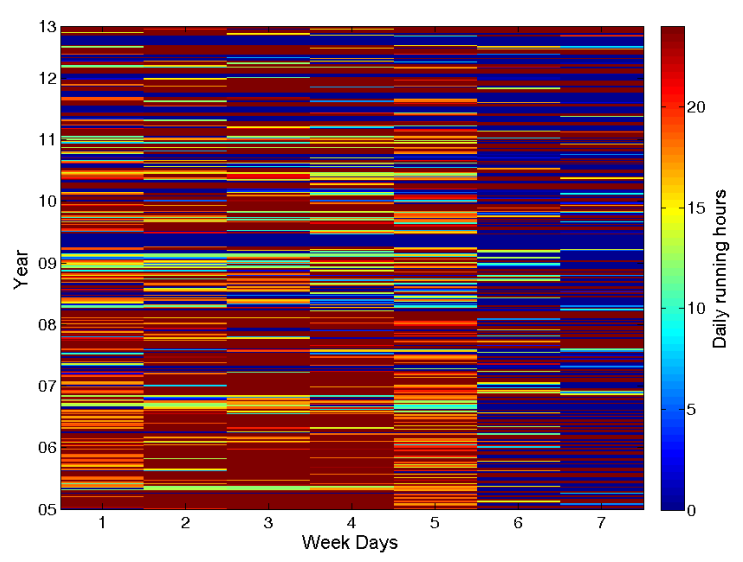

(a)

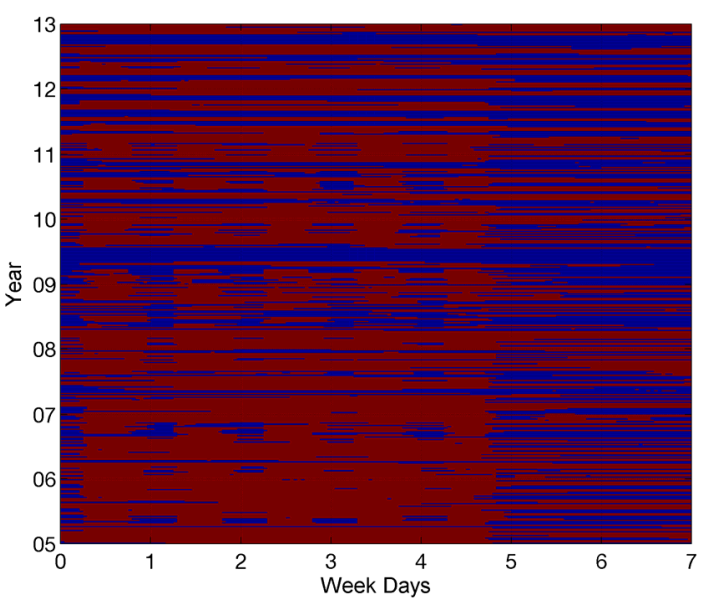

(b)

Fig. 4. Image representation of the weekly operational patterns: (a) daily running hours; (b) binary notation of running hours

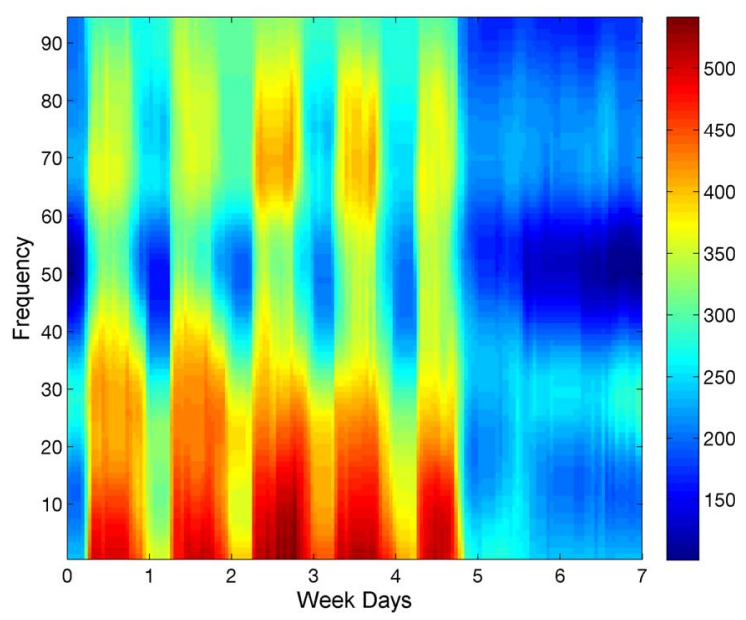

Fig. 5. Spectrogram of the weekly pattern matrix. 


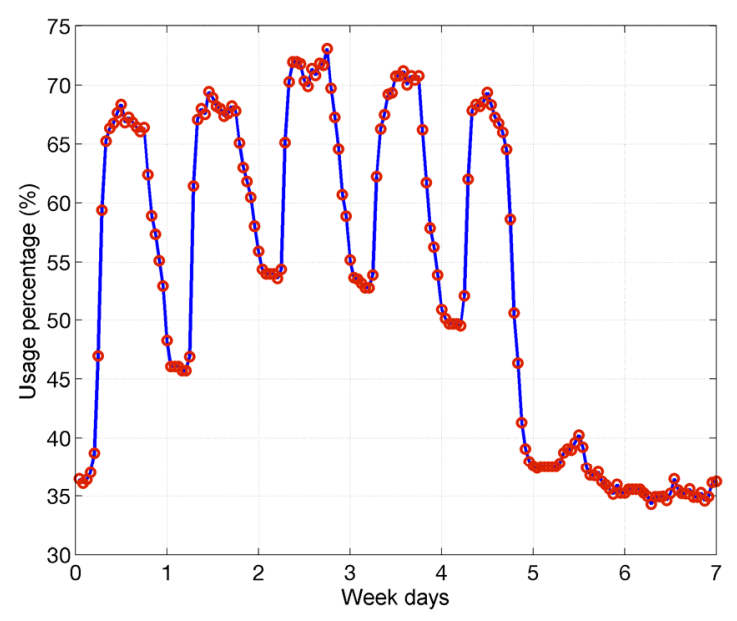

(a)

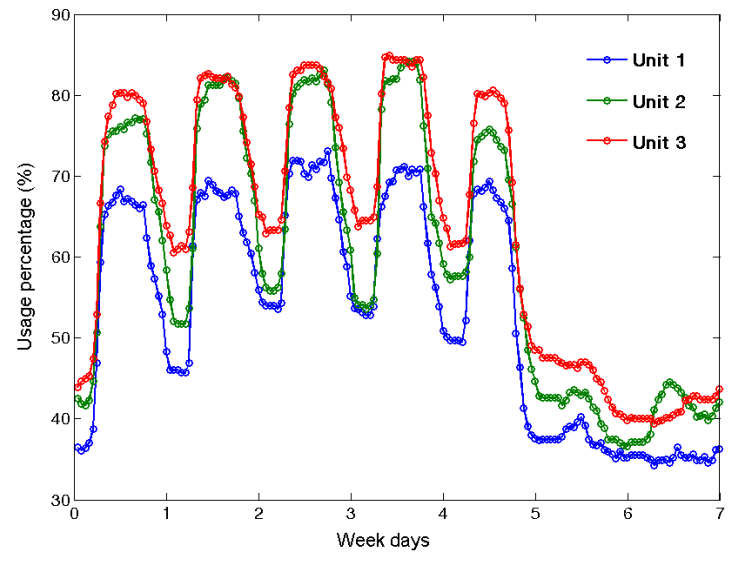

(b)

Fig. 6. Mean percentage usage: (a) of the studied unit; (b) comparing three units

\section{E. Trend analysis}

Taking the spectrogram of Fig. 4(b) with respect to annual $(\times 52$ week periods) and calculating the weekly mean percentage usage (similar methodology as in Section D), gives the results shown in Fig. 7 (blue), from which the operational 'trend' of mean usage is obtained through the application of SSA, as shown in Fig. 7(a).

From the results, it can be seen that a significant increase in winter usage regularly exists (northern hemisphere) which can be used for forecasting operational behaviour in advance. For instance, forecast of usage percentage for one year in advance is shown in Fig. 7(b); again providing extremely valuable input for preventive maintenance scheduling.

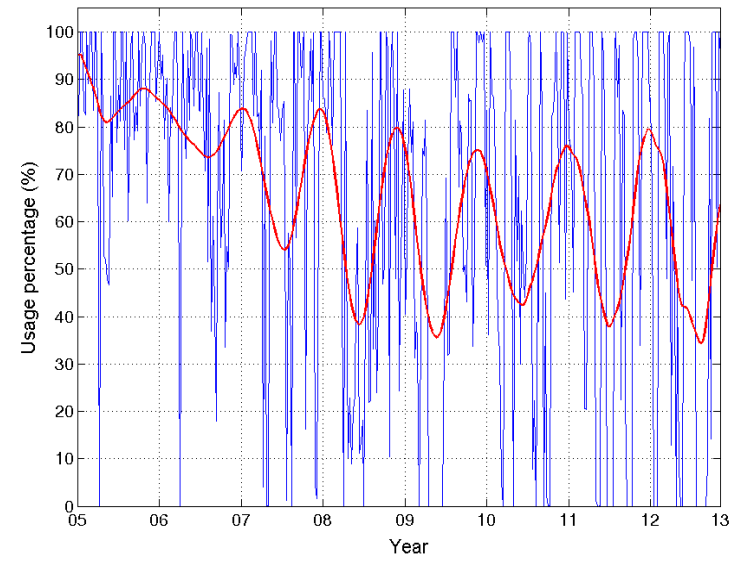

(a)

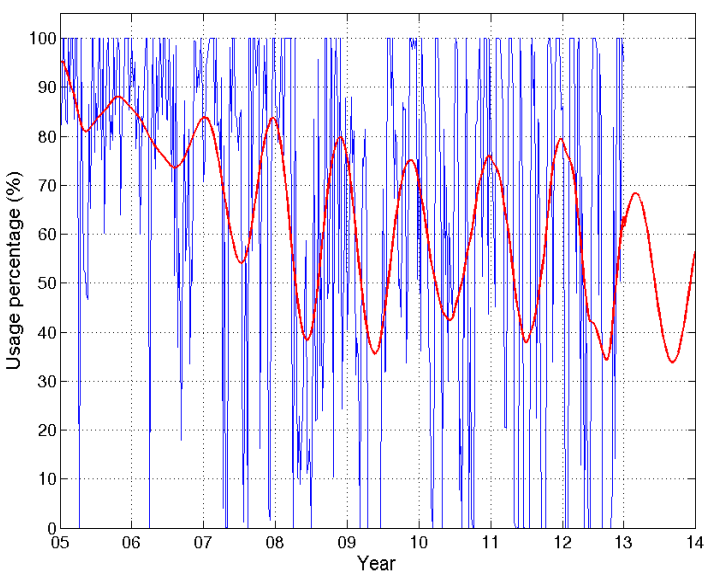

(b)

Fig. 7. Average usage percentage wrt the yearly weeks: (a) trend extraction (red); (b) forecasting

\section{CONCLUSION}

The paper has considered techniques for the extraction of operational behaviour patterns for industrial systems, with an industrial gas turbine being used to focus the study and highlight the benefits. Through use of experimental trials it has been shown that both the users startup behaviours and weekly and annual patterns of duty can be readily extracted, and be further used for forecasting future behaviour. In this way, the primary characteristics that influence predictive maintenance scheduling to enhance performance and reduce operational downtime have been extracted and identified. It should be noted that whilst this study has focused on inductrial gas turbines, the underlying principles are readily applied to other industrial systems.

\section{ACKNOWLEDGMENT}

The authors would like to thank Siemens Industrial Turbomachinery, Lincoln, U.K., for providing research support and access to real-time data to support the research outcomes. 


\section{REFERENCES}

[1] V. Thipsuwanporn, A. Numsamran, M. Leawsoong. Balance weight fault detection in compressor using FFT algorithm. In 12th Int. Conf. on Control, automation and systems (ICCAS), pp. 798-802, 2012.

[2] D. Liu, J. Tian, B. Yang, J. Sun. Time-Frequency Analysis Based Motor Fault Detection Using Deconvolutive STFT Spectrogram. Journal of Convergence Information Technology, 7(8): 295. 2012.

[3] Z. Yang, C. Bingham, B.W.K. Ling, Y. Zhang, M. Gallimore, J. Stewart. Unit Operational Pattern Analysis and Forecasting Using EMD and SSA for Industrial Systems. Advances in Intelligent Data Analysis XI , Lecture Notes in Computer Science, 7619: 416-423. 2012.
[4] E.O. Brigham. The Fast Fourier Transform. New York: Prentice-Hall. 2002.

[5] A.V. Oppenheim, R.W. Schafer. Discrete-time signal processing. Prentice-Hall, Englewood Cliffs, NJ, 1989.

[6] N. Golyandina, V. Nekrutkin, A. Zhigljavsky. Analysis of Time Series Structure: SSA and related techniques. Chapman and Hall/CRC, New York. 2001. 